\title{
Après la fête : la sphère privée du chant chez les Amhara d'Éthiopie
}

\section{Katell Morand}

\section{OpenEdition}

1 Journals

Édition électronique

URL : https://journals.openedition.org/clo/2328

DOI : $10.4000 /$ clo.2328

ISSN : 2266-1816

Éditeur

INALCO

\section{Édition imprimée}

Date de publication : 31 décembre 2015

ISBN : 9782858312276

ISSN : 0396-891X

\section{Référence électronique}

Katell Morand, «Après la fête : la sphère privée du chant chez les Amhara d'Éthiopie », Cahiers de littérature orale [En ligne], 77-78 | 2015, mis en ligne le 10 mai 2016, consulté le 30 juin 2021. URL: http://journals.openedition.org/clo/2328 ; DOl : https://doi.org/10.4000/clo.2328

\section{(@) $(1) \Theta$}

Cahiers de littérature orale est mis à disposition selon les termes de la Licence Creative Commons Attribution - Pas d'Utilisation Commerciale 4.0 International. 


\title{
Après la fête : la sphère privée du chant chez les Amhara d'Éthiopie
}

\author{
Katell Morand \\ Lesc, Paris Ouest Nanterre la Défense
}

Note de la rédaction : l'amharique possède sa propre écriture, pour faciliter la lecture, tous les textes sont ici présentés dans une transcription en alphabet latin qui suit le système choisi par Kane (1990).

La conversation avait cessé depuis de longues minutes déjà lorsque Gätənnät se mit à chanter. Il était tard et les vaches, en quête de chaleur, se pressaient les unes contre les autres. Dans la minuscule clairière où nous campions depuis la veille, la voûte basse de la végétation masquait le rayonnement de la lune, laissant le feu pour unique source de lumière. Après l'avoir alimenté, son jeune frère s'était enroulé dans sa pièce de coton puis couché dans le coin opposé de l'enclos, où l'on distinguait à peine sa silhouette immobile. Gätənnät, légèrement penché en avant et la main collée contre l'oreille, chantait d'une voix claire :

Awäy yäsaw nägär yabalañ wägän antä Hélas, la condition humaine, je suis triste pour toi, mon compagnon,

Motənəm bətəmot käman təbältalähə

Tu es mort, certes, mais tu ne surpasses personne,

Ondäw am ${ }^{w}$ am ${ }^{w}$ atวh tasazənänalähə

C'est par la façon dont tu es mort, que tu me peines.

Il poursuivit quelques minutes avant de s'arrêter aussi abruptement qu'il avait commencé. Autour de lui, rien ne bougeait ; son frère dormait toujours. 
Gätənnät était un chanteur très apprécié de la petite communauté paysanne des hauts plateaux amhara du Goğğam, dans le nord de l'Éthiopie où j'effectuais alors mes recherches ${ }^{1}$. J'avais pu le constater moins d'une semaine auparavant, le soir de la fête annuelle du saint de la paroisse ${ }^{2}$, alors qu'un petit groupe de ses invités, très animés et encore un peu ivres, rentraient chez eux après la nuit tombée. Après quelques commentaires élogieux sur la voix et le talent poétique de leur hôte, ils avaient rappelé la tension d'un certain moment qui heureusement n'avait pas duré : ni bagarres à coup de bâton ni fusils imprudemment brandis, toutes choses qui arrivent parfois lorsqu'on se lance dans le chant. Ce soir-ci, en revanche, ce n'était pas pour divertir ou impressionner un public mais « pour lui-même » (läbə̌č́a) qu'il chantait. Comme tous les autres paysans de la région, hommes ou femmes, il meublait chacun de ses instants solitaires ou « privés » (yägal) de poèmes chantés - une pratique dont les intéressés parlent peu, mais qui représente une part si importante de leur vie musicale qu'elle mérite qu'on y prête attention. Cet article a donc pour objet de mieux comprendre la sphère privée du chant en s'attachant plus particulièrement au discours et au statut de l'énonciation, ce qu'il s'y dit et pour quelles raisons.

\section{Soi-même pour tout public ?}

Il peut paraître paradoxal de considérer la solitude comme un objet d'étude pour l'anthropologie ou les sciences sociales en général. Celles-ci se préoccupent des interactions et des échanges (y compris symboliques) qui sont au cœur de la vie sociale et des pratiques culturelles. L'ethnomusicologie nous a montré la richesse et les enjeux de la pratique musicale : rituels ou « chants de compagnie $»^{3} s^{\text {'éclairent }}$ grâce à l'analyse acoustique, l'interprétation anthropologique et la pragmatique (c'est-à-dire l'étude en contexte des performances musicales). Il faut dire que la solitude, en tant qu'envers de la sociabilité, est par extension souvent considérée comme le domaine souverain de l'individu et donc en dehors du monde social. De là viendrait d'ailleurs sa fécondité potentielle, offerte au génie créateur, poète ou musicien, dont elle nourrirait l'inspiration. Le regard occidental est façonné par cette conception romantique de la solitude comme refuge, retrait, isolement

1. Cet article s'appuie sur un total de vingt-et-un mois de séjour dans le Goğğam entre 2003 et 2013.

2. Les paysans du Goğğam sont dans leur grande majorité des chrétiens orthodoxes de l'Église éthiopienne.

3. Bernard LORTAT-JACOB, 1998. 
des semblables : celle qui permet au Rousseau des Rêveries du promeneur solitaire d'être « détaché de tout » pour, pleinement lui-même, « converser avec [son] âme $\gg^{4}$.

Bien que la solitude soit fertile, on pourrait penser que la musique, à parcourir les rares textes qui lui sont consacrés, y est un phénomène plutôt rare. Au-delà des difficultés indéniables de l'enquête ethnographique, l'existence même de performances solitaires est une énigme : comment imaginer un chant qui ne serait pas destiné à être entendu ? Qui ne communiquerait rien ${ }^{5}$ ?

Pierre Clastres est l'un des rares ethnologues à avoir décrit une performance musicale « pour soi ». Dans le cinquième chapitre de la Société contre l'État, intitulé « L'arc et le panier » (1974), il introduit son analyse de la structure des échanges chez les Guayaki par une description frappante des chants nocturnes des chasseurs. Il s'attarde sur la nuit qui envahit la forêt, montre le sommeil qui gagne les campeurs, puis se tourne vers les chasseurs silencieux :

Ils ne dorment pas cependant et leur regard pensif, retenu aux ténèbres voisines, montre une attente rêveuse. Car les hommes s'apprêtent à chanter et ce soir, comme parfois à cette heure propice, ils vont entonner, chacun pour soi, le chant des chasseurs : leur méditation précède l'accord subtil d'une âme et d'un instant aux paroles qui vont le dire. Une voix bientôt s'élève, presque imperceptible d'abord, tant elle naît intérieure, murmure prudent qui n'articule rien encore de se vouer avec patience à la quête d'un ton et d'un discours exact. Mais elle monte peu à peu, le chanteur est désormais sûr de lui et soudain, éclatant, libre et tendu, son chant jaillit. Stimulée, une seconde voix se joint à la première, puis une autre ; elles jettent des paroles hâtives, comme réponses à des questions qu'elles devanceraient toujours. Les hommes chantent tous maintenant. Ils sont toujours immobiles, le regard un peu plus perdu ; ils chantent tous ensemble, mais chacun chante son propre chant. Ils sont maîtres de la nuit et chacun s'y veut maître de soi ${ }^{6}$.

4. Jean-Jacques Rousseau, 1983 [1778], p. 27.

5. « Car peut-on imaginer une musique destinée à ne pas être entendue ? De la musique pour rien ? [...] en tant que produit de l'oralité destiné à l'oralité, la musique n'a pas de réelle existence en dehors des circuits et relais qui permettent sa production et sa transmission. » (LORTAT-JACOB, 2002, p. 458-459)

6. Pierre Clastres, 1974, p. 88-89. 
Ces hommes chantent donc à proximité les uns des autres, mais sans s'écouter ni se coordonner. Ce paradoxe amène l'auteur à questionner le statut sémiologique de ces chants, entités autonomes n'existant qu'à la seule intention du musicien qui l'énonce dans un «impossible tête à tête avec soi ». Que devient une parole lorsqu'elle cesse d'être un outil de communication, lorsqu'elle est « détournée de sa fin "naturelle", qui est la relation à l'autre $»^{7}$ ? L'absence de destinataire briserait le schéma de communication fondé sur la transmission d'un message d'un émetteur à un récepteur. Chanter pour soi équivaudrait à une circularité de l'énonciation et donc à une négation de cet autre échange fondateur qu'est le langage comme communication de signes linguistiques - un « endo-langage », qui serait en définitive la « négation de tout message ». L'interprétation que P. Clastres propose de ces chants est donc directement liée au statut (ou plutôt, non-statut) sémiologique qu'il leur assigne. Langage affranchi des contraintes du signe et de la communication, le chant solitaire des hommes serait l'échappatoire à l'échange qui fonderait la société Guayaki tout en emprisonnant les individus.

Cette logique n'est évidemment pas sans poser quelques problèmes. Le texte de P. Clastres est parsemé d'expressions relevant du champ des interactions, qui sont autant d'indices que quelque chose est bien communiqué : les paroles « disent », les chanteurs sont à la recherche « d'un ton et d'un discours exact », une voix est «stimulée » par une autre. Tous ces paradoxes proviennent en réalité d'une conception un peu rigide du schéma de communication et des positions respectives de l'émetteur qui code le message, et du récepteur qui le décode. Or il n'est pas démontré qu'une musique ou qu'une parole soit sans signification dès lors qu'aucun destinataire n'est identifiable. Ce dernier peut être invisible, ou même imaginaire. La musique joue ainsi un rôle crucial dans certains rituels qui font intervenir des êtres invisibles au cours d'interactions aux dynamiques complexes (Rouget, 1980). L'adresse et le dialogue peuvent aussi devenir intra-individuels, comme dans le cas des ànent, ces chants privés des Jivaro Achuar analysés par Anne-Christine Taylor et Ernesto Chau (1983). «Invocation » à des êtres surnaturels, ces chants possèderaient un caractère à la fois secret et magique. Cette idée de la musique privée comme vecteur privilégié du dialogue avec l'invisible se retrouve également dans les travaux de Stéphanie Weisser (2008) sur le bägäna, la grande lyre amhara qu'elle définit comme un instrument à la fois de médiation avec le divin et de dialogue avec soi-même.

À qui s'adresse ici Gätannät dans son chant solitaire ? À lui-même, à une personne imaginaire, à un être invisible ? Pour tenter de répondre à ces questions,

7. Pierre Clastres, 1974, p. 106. 
il faut d'abord comprendre ce que signifie la notion de chant « privé » pour les Amhara et ce qu'elle recouvre.

\section{Du public au privé : le lien musical}

\section{Des performances à part?}

À première vue, le chant en solitude se situe radicalement à part dans l'univers des performances musicales chez les Amhara. Ces dernières surviennent au cours de rassemblements qui sont le plus souvent soumis au calendrier religieux ou agricole, et toujours régis par la parenté, c'est-à-dire par le principe d'entraide et de réciprocité qui fonde les relations entre zämäd, ces individus qui se reconnaissent une connexion généalogique ${ }^{8}$. Selon le type d'événement, les cercles plus ou moins larges des parents mobilisés définissent différents niveaux de sociabilité, depuis les commémorations funéraires (qui peuvent réunir plusieurs centaines de personnes) jusqu'aux petites fêtes intimes et impromptues qui sont offertes au cousin de passage.

La musique « pour soi » se définit par des temps et des espaces différents. Elle ne surgit que dans les interstices de la vie sociale : durant les travaux solitaires, comme la garde des troupeaux ou le puisage de l'eau, ainsi que dans les lieux en marge du village, comme cette « nature sauvage » (dur) dangereuse et inquiétante, où les hommes emmènent leur bétail et d'où ils ne rentrent parfois qu'au bout de longues semaines ${ }^{9}$. Ce n'est qu'une fois dans cet isolement complet, me disent ainsi mes interlocuteurs, « qu'il n'y a que les arbres, la forêt et les animaux sauvages $\gg^{10}$, qu'ils se mettent à chanter. Élusifs par définition et impossibles à observer, ces chants se laissent parfois surprendre au détour d'un sentier.

Si l'isolement complet représente, pour ainsi dire, la forme canonique du privé, il n'en est cependant pas la règle. Gätənnät, après tout, chante à proximité de son frère : qui sait si ce dernier dort vraiment ? Que penser de la présence incongrue de l'ethnologue et de cet enregistrement que, le lendemain, il ne manquera pas d'écouter ? Et des femmes qui, lorsqu'elles se rendent au fleuve en petits groupes, feignent de ne pas entendre celle qui fredonne ? Il s'agit là de formes particulières d'interactions, où le concept de « solitude » (bəčannät) semble acquérir une acception un peu plus large, et recouvrir ce que j'appellerai des solitudes accompagnées : des moments où l'on peut être seul à plusieurs et le chant conserve son caractère privé.

8. La parenté chez les Amhara est bilatérale (НовEN, 1973).

9. Pour une analyse des représentations associées à la forêt, voir MoRAnd (2013).

10. Abäba Tasaw, novembre 2006. 
Cette solitude en compagnie est une des caractéristiques du bärät, cet enclos en forêt ou en plein champ où le bétail est gardé de nuit. Tous les soirs de saison sèche, hommes et jeunes garçons s'y retrouvent en un petit groupe provenant d'une même maison ou de maisons voisines (frères ou pères avec leurs fils). Comme le montre l'exemple de Gätənnät, celui qui chante « pour soi » et rompt le silence a alors parfaitement conscience de la présence des autres : mais il ne peut savoir si ces derniers dorment ou écoutent dans l'obscurité et ne semble pas s'en préoccuper. Les enfants de six ou sept ans qui dorment à l'occasion dans la même couverture que leur père apprennent ainsi très tôt à respecter cette entente tacite et s'abstiennent de regarder ou de réagir.

Car tous les participants du bärät ne sont pas également susceptibles de chanter. Seul le plus âgé d'entre eux, le père ou le frère aîné, peut se considérer comme «seul » dans une telle situation. L'âge joue un rôle très important, non pas dans l'absolu, mais dans la différence de statut qu'il produit entre les individus, entre celui qui est responsable d'un foyer et celui qui travaille sous son autorité ou ne s'en est pas totalement affranchi. Toutefois, plus qu'à la crainte ou au respect de leurs aînés, le silence des plus jeunes semble dû au plaisir intense qu'ils éprouvent à les écouter. Les nuits passées ensemble dans le froid, à veiller contre les hyènes et les voleurs, ainsi que la voix du père, du frère ou de l'oncle s'élevant tout près d'eux sont l'un des symboles de la relation toute particulière qui les unit. La solitude accompagnée se définit donc par une extension temporaire de la personne solitaire à un cercle de personnes très précises. Le chant émerge d'un silence confortable, en est le prolongement et en serait presque un équivalent puisqu'il s'agit, pour l'individu, de signifier le repli sur soi-même tout en niant l'existence d'une quelconque communication.

En dehors des visites qu'elles se rendent, les femmes connaissent elles aussi de longs moments de solitude accompagnée, lorsqu'elles travaillent au jardin ou filent leur coton par exemple. Pour elles également, certaines conditions doivent être réunies. Les femmes ne chantent que devant celles qui partagent leur toit ou l'ont partagé dans le passé (autrement dit leurs mères, sœurs, et filles), jamais devant les hommes de leur foyer ${ }^{11}$. Mais alors que ces derniers bénéficient de l'obscurité, des arbres, et d'une relative distance physique pour établir le privé, la solitude accompagnée des femmes ne peut être maintenue, en plein jour et à une distance

11. Et bien entendu, jamais devant des invités, ce qui a limité à mon hôtesse et quelques voisines mes possibilités d'entendre de tels chants. Les difficultés rencontrées lors de l'enquête ethnographique et de la constitution d'un corpus sont analysées plus en détail par Morand (2008). 
minimale - parfois inférieure à un mètre - que par d'autres indices, plus ou moins subtils : une voix dont le volume ne dépasse guère le murmure, une concentration ostensible sur le travail, des regards qui s'évitent ou encore le repli d'un pan de toge sur la bouche, à la manière dont les personnes malades ou fatiguées se retirent de la conversation. Et il suffit souvent de quelques mots à peine pour ramener la chanteuse dans la conversation et la sociabilité.

\section{Que chantent-ils?}

La question de la place de ces chants solitaires dans les pratiques musicales des Amhara se complique lorsqu'on se penche plus précisément sur ce qu'ils chantent.

Certaines cultures ont une tradition poétique de la solitude. C'est le cas, par exemple, des Touaregs et de leur esuf décrit par Dominique Casajus (2000). Souvent traduit par «solitude », esuf désignerait en effet les espaces sauvages que traversent les hommes lors de leurs voyages mais aussi l'état d'esprit qui s'empare d'une personne lorsqu'elle se retrouve seule ou confrontée à l'adversité (Casajus, 2000, p. 69) : un « sentiment doux-amer » de mélancolie ou d'angoisse, provoqué par l'absence de l'être aimé, la tente abandonnée, les déserts silencieux, sont aussi des figures obligées par lesquelles le poète représente le processus de composition de son poème.

Bien que les paysans Goğğamés se montrent très élusifs dans les commentaires de leurs performances « privées », j'ai noté que quelques mots revenaient systématiquement lors de nos discussions. Il s'agissait de mots d'émotion, comme la « joie » (dästa), l'《angoisse » (čnqü̈t) ou la « colère » (nəddet), et du terme təzəta, que la plupart des dictionnaires traduisent par « souvenir », « remémoration » ou « réminiscence » (Baeteman, 1929; Kane, 1990) mais qui ne semble pas avoir de véritable équivalent dans les langues européennes. Tazəta est ce qui « vient » dans la solitude, comme me l'expliqua mon hôtesse, Abäba, quelques jours avant la célébration de l'une des fêtes majeures du village. Elle rentrait alors du fleuve où elle était partie puiser l'eau. Une fois seule au fleuve me dit-elle, la təzəta lui était venue du temps où ils étaient riches : elle se faisait à présent du souci pour la nourriture qu'elle pourrait offrir à ses invités, mais à l'époque elle disposait d'assez de viande et d'œufs pour tout le monde. Alors elle s'était assise là, conclutelle, à pleurer et à chanter au bord de l'eau.

La təzəta semble être un état d'esprit particulier, associé à la comparaison avec le passé et à l'absence - ce qui n'est pas ou n'est plus là - comme si le vide créé par la solitude était comblé par des pensées intrusives. La tazata serait-elle donc un équivalent de l'esuf? La question est plus complexe qu'il n'y paraît, car le terme est très chargé de sens dans l'Éthiopie contemporaine. Profondément lié à la production 
musicale moderne en langue amharique - c'est-à-dire celle qui s'est développée en milieu urbain à partir des années 1960 - il désignerait selon certains auteurs une forme de «blues éthiopien » et semble même avoir atteint le statut de sentiment national, en particulier auprès de la diaspora (Ashenafi Kebede, 1971 ; Falceto, 2004 ; Delombera Negga, 2004). Beaucoup considèrent que cette figure littéraire et l'esthétique musicale qui l'accompagne viendraient en ligne directe de la musique traditionnelle des Haut-Plateaux et plus particulièrement d'une chanson éponyme dont pourtant, comme le remarquent S. Weisser et Francis Falceto (2013), aucune trace n'existe avant les années 1960.

De fait, dans les campagnes du Goğğam, la tazəta n'est connue ni comme titre de chanson, ni comme figure littéraire ni même comme répertoire, pour la bonne raison qu'il n'y existe pas de chant de solitude à proprement parler. Ce que les paysans Goğğamés chantent en privé est exactement « la même chose », disentils, que ce qu'ils auraient pu chanter ou entendre quelques mois auparavant lors d'une moisson collective, par exemple. Que cela signifie-t-il donc ?

Avant toute discussion, il faut souligner qu'il n'y a pas, chez les Amhara, d'équivalent strict à nos notions de «musique » ou de « chant ». Ce que, en tant qu'Occidentaux, nous entendons et classons intuitivement comme « musique » se répartit en réalité en un petit nombre de catégories discrètes et mutuellement exclusives que, par commodité, j'appelle des genres. En sociabilité comme en privé, les Goğğamés pratiquent ainsi trois genres principaux de poésie chantée : le qärärto, l'angurguro et le zäfän. À chacun de ces genres correspond une action différente, désignée par un verbe spécifique : ainsi, on dit d'une personne qu'elle « fait du qärärto » (aqrara), qu'elle « fait de l'ongurguro » (angüräggürä), qu'elle « fait du zäfän » (zäffänä). Ces genres n'appartiennent cependant pas à des domaines entièrement séparés. Tous mettent en jeu :

- dəmș, la voix ;

- un zema, mélodie ou contour mélodique ;

- un gətəm, c'est-à-dire un poème, et plus précisément le distique rimé qui est au cœur de la poésie de langue amharique ${ }^{12}$.

Ces genres appartiennent en effet à un champ plus vaste, désigné par le concept de « dire » ou d' « énoncer » (alä), qui regroupe donc aussi bien la parole que le chant. Il est possible, à la place du verbe spécifique, d'employer les expressions « dire du qürärto » (qürärto alä) ou « dire du zäfän » (zäfän alä), par exemple. Il serait donc plus judicieux de parler de musique-poésie tant ces deux aspects

12. Pour la construction du distique amharique, on se réfèrera à CoHen (1924) et Menghistu Lemma (1963). 
sont mêlés et tant leur séparation mène à la confusion, ce qui constitue l'un des principaux problèmes rencontrés dans la littérature éthiopisante ${ }^{13}$.

Un deuxième problème concerne la définition de ces genres : les critères les plus souvent retenus sont le contexte de performance, le statut des chanteurs ou la thématique des chants; ce qui mène à des catégories telles que « chants de travail » ou « de mariage », « chant de bergers » ou « de guerriers », « chants de femmes », etc. Ces catégories sont rarement les mêmes d'un auteur à l'autre : l'angurguro, par exemple, est un « chant de solitude » pour les uns (Levine, 1965, p. 270 ; Ashenafi Kebede, 1971, p. 64-65), mais une « complainte à l'occasion d'un deuil » pour d'autres (Mondon-Vidailhet, 2003). De plus, comme le monde amhara est loin d'être uniforme et que les appellations varient d'une région à l'autre, on retrouve différentes définitions pour des mêmes genres ou des classifications identiques pour des genres différents.

Ce que cachent ces tentatives de classification est que les genres peuvent, en réalité, être tous pratiqués dans une grande variété de circonstances et par toutes sortes d'individus, indépendamment du sexe et de l'occupation. Qu'il s'agisse d'un rituel ou d'une petite réunion entre parents proches, les mêmes genres sont sollicités ${ }^{14}$. Le qärärto, par exemple, apparaît aussi bien lors de fêtes religieuses, de mariages, de moissons collectives, ou d'enterrements. Statistiquement il serait plutôt l'affaire des hommes, comme l'affirme Getie Gelaye (2000), mais de nombreuses femmes le pratiquent également. L'angurguro, souvent associé aux rituels funéraires, peut aussi se chanter pendant le travail ou seul en forêt. Autrement dit, sphères publiques et privées font appel aux mêmes genres.

Plus encore, elles font appel à de mêmes chants et à de mêmes poèmes : ce que les Goğğamés chantent en privé n'est jamais une composition originale mais un chant qui a déjà fait son apparition quelques mois, quelques années, voire quelques décennies auparavant, dans le cadre de la sociabilité. Cela peut paraître surprenant compte tenu de l'importance de l'improvisation poétique dans tous les contextes de sociabilité, aussi bien rituels que plus intimes. Pourquoi chanter une seconde fois, dans la solitude, quelque chose qui l'a déjà été ? Quels rapports entretiennent les chants privés avec les performances publiques, et que signifie le terme de tazata?

13. Les mêmes genres y sont présentés tantôt comme musique (Ashenafi Kebede, 1971), tantôt comme poésie (Cohen, 1924 ; Getie Gelaye, 2000).

14. À l'exception, bien entendue, de la musique liturgique, réservée aux prêtres. 
CAHIERS DE LITTÉRATURE ORALE

82 Paroles publiques, paroles confidentielles $-\mathrm{n}^{\circ} 77-78$

\section{L'écho de la fête, ou le social dans la solitude}

\section{Des chants venus du passé}

Une anecdote devrait nous aider à mieux comprendre. L'une des femmes avec lesquelles il m'a été possible de travailler sur le chant en solitude était une voisine de mes hôtes nommée Haymanot. Il m'arrivait souvent d'entendre sa voix s'élever depuis sa cour, à quelques dizaines de mètres de la nôtre. Un jour, attirée par des bruits de voix, je la trouvai à la porte de son étable. Sanglotant et chantant tout à la fois, elle y battait le beurre de façon désordonnée : «C'est sur ma vie que je pleure », lança-t-elle en m'apercevant, avant de reprendre sa mélodie cahotée : « Hélas mon père, mon monde... ». Elle m’entraîna dans la maison sans cesser de chanter ni d'agiter nerveusement ses bras, renversant au passage les calebasses qu'elle tentait de ranger. «Les soucis me sont trop nombreux », finit-elle par dire après s'être assise sur sa banquette de terre, au ras du sol. Se calmant peu à peu, elle continua à chanter en égrenant son coton :

Dämomə abbat aläm dämom wändəm Et aussi mon père mon monde, et aussi alämə mon frère mon monde,

Antäna andet largaho

And gaze mutähr and gize tänässa

Yäləğočahəmə nägär aytə hidənna
Que puis-je faire de toi ?

Tu es mort une fois, lève-toi une fois,

Regarde les problèmes de tes enfants, et puis va-t'en.

Elle fit une pause. Elle pleurait tout le temps, me dit-elle, car elle n'avait plus de famille depuis que son frère et son père étaient morts (tous deux furent tués par balle); et personne pour la protéger. Ainsi toujours cette tazəta, conclut-elle, lui venait dans ses instants de solitude.

Or elle ne chanta pas n'importe quel chant ce jour-là : ce qui lui était « venu » dans sa solitude était un əngurguro qui avait été originellement composé et performé lors de l'enterrement de son père, probablement par un membre de sa parentèle. Car les poèmes chantés dans la solitude sont toujours associés aux personnes précises qui les énoncèrent originellement où auxquelles ils étaient adressés : un parent décédé ou qui vit loin, auquel la musique redonne présence le temps d'une performance. Comme me l'expliqua l'un des cousins d'Haymanot : « Tu entends quelqu'un faire du qärärto, ton frère par exemple. Quand il disparaît, qu'il est 
mort ou qu'il est parti, tu en as la təzəta en refaisant le même qärärto ${ }^{15}$. Pour ce jeune homme, il s'agissait de son père, alors en prison, et dont il m'affirmait voir l'image et entendre la voix avec une vive acuité dès qu'il se mettait à fredonner - ou simplement siffler - les qärärto que son père avait lancés d'une voix puissante une nuit qu'ils passaient tous deux en forêt. Il le voyait debout, tenant son fusil « comme un chasseur », « c'est comme s'il était là, je me sens heureux ${ }^{16}$.

Revoir des proches en imagination : c'est aussi ce qui arrivait à Getačaw, un autre jeune homme qui devait avoir dix-huit ou dix-neuf ans et était venu d'un autre district du Goğğam pour garder le bétail d'un paysan aisé du coin. Je le rencontrais régulièrement dans les pâturages au bord du fleuve et il acceptait, de temps à autre, de me commenter ses performances chantées. Un jour, alors qu'il venait de terminer deux séries de qärärto, je lui demandai ainsi s'il était capable de détailler les « images » qu'il me disait avoir en tête. Les descriptions qu'il m'en donna reflètent, avec beaucoup d'économie, la vivacité ainsi que la précision visuelle, sonore et même kinesthésique des scènes remémorées. La première série était « de la joie, la təzəta d'un ami, à l'enclos aux vaches de [son] père », m'expliqua Getačaw, en montrant d'un geste de la main où se trouvaient les veaux et chacun des hommes endormis par rapport à lui. Quant à la deuxième série, il s'agissait de la tazəta d'un homme âgé, nommé Mulat, qui entonnait lors d'une joute chantée le distique ci-dessous :

Olm kalläw čakka alm kalläw dur

Dans la forêt profonde, dans la nature profonde,

Siyaguärämärämäw yadära läsäw ondä Hommes comme léopards, ils rugissent näbar ceux qui y passent la nuit.

C'était lors d'une moisson et, dans son souvenir, « il y a de la bière, du maïs bouilli, de l'anğära, du lait fermenté et de l'hydromel ${ }^{17}$. Une fois à la maison, le café est bouilli. Les moissonneurs sont quinze, ils font le qürärto en se le passant à tour de rôle. Il y a du soleil et il fait chaud. Le champ de teff, c'est celui de mon oncle $\gg^{18}$.

15. Sisay Fasigaw, février 2007.

16. Sisay Fasigaw, novembre 2006.

17. L' ənğära est la galette de teff (une céréale endémique) à la base de l'alimentation dans les hauts plateaux du nord de l'Éthiopie.

18. Getačaw Assäfa, janvier 2006. 
Il est frappant que les images décrites par mes interlocuteurs représentent toutes des scènes d'interaction sociale, avec leurs règles et leur part de spontanéité. Toutes donnent le sentiment que quelque chose de particulier s'était dit entre les individus présents, un quelque chose sur lequel nul ne s'étend mais qui semble devoir être délibérément recréé ou réinterprété à chaque itération solitaire du chant.

\section{Citations musicales : rejouer l'interaction}

Il existe un terme pour désigner la répétition volontaire de fragments de discours : la citation. De celle-ci nous faisons tous régulièrement l'expérience, à l'écrit comme à l'oral, lorsque nous nous prenons comme argument des mots d'un auteur célèbre ou ceux d'une de nos connaissances. Or qu'est-ce que citer ? C'est « se faire l'écho des mots et des voix d'autres personnes », comme l'écrit Ruth Finnegan dans le livre qu'elle y consacre, et dans lequel elle partage certaines de ses propres expériences :

I recall words and voices from my younger days and at times still use them with other family members today. We quote my father's "If you want something done ask a busy man", words I still hear in his voice and have always taken as his own - not originated by him, I now realize, but anyway we pass them on in his name. Still mimicked with affectionate amusement is my father's reproachful "Now dear..." (in a tone which conveyed anything but endearment). ${ }^{19}$

En effet, il s'agit bien de rappeler une voix : les mots prononcés bien sûr, mais aussi la mélodie de l'intonation et l'expressivité du visage. En citant une voix, qu'elle soit celle d'un autre ou la sienne propre (avec toute la distance qu'implique le temps écoulé), c'est en réalité une situation de communication qui est ravivée, ses interlocuteurs et leurs intentions qui sont rappelés, et tout son contexte qui est réactualisé. Le « Now dear ... » du père de R. Finnegan dit quelque chose de ce qu'était leur relation : il en est une facette, rappelée à un moment opportun avec humour et tendresse, tout autant (et probablement plus) pour soi-même que pour son interlocuteur. Ne pourrait-on donc penser que les chants solitaires des Goğğamés consistent en des citations chantées ravivant d'anciennes situations de performances avec leurs interactions, leurs contextes, et leurs significations ?

Mes interlocuteurs semblaient avoir une difficulté, ou peut-être une réticence, à répondre à la question de leur choix : ils avaient tous, comme je pouvais le constater, quantité de souvenirs musicaux concernant les mêmes individus ou des

19. Ruth Finnegan, 2011, p. 9. 
situations très similaires. Pourquoi avaient-ils préféré tel chant à un autre en ce moment précis ? Existait-il une raison ? Beaucoup mettaient en avant le choix du genre («c'est un qärärto ») et le contexte de la performance rappelée («c'était la fête du saint, tu sais bien ce que je veux dire »). Ce qui revenait le plus souvent, cependant, était l'importance du «sens » $\left(f_{\partial} \check{c} c\right)$ du poème. Or déceler ce sens n'est pas toujours une tâche aisée : même lorsque les poèmes ne recourent pas à la célèbre technique du «wax and gold », le style proverbial, elliptique, et ponctué de métaphores ou de jeux de mots les rend assez difficiles à interpréter ${ }^{20}$. Leurs propos s'avèrent très contextuels, comme le qürärto ci-dessous que Gätənnät accepta un soir de commenter :

Man Tăga natə man kafu kabtə nat Quel veau est-ce, quel méchant bétail čärqen yäbälačäw est-ce, qui a mangé mes vêtements,

Yäbwalaw nägär azura bayäc̈äw

Sans en avoir envisagé les conséquences!

« Ce qärärto, c'est la tazata d'une moisson collective l'année dernière », me dit-il. C'est lui-même qui l'avait chanté. Son frère (qui cette fois-ci encore dormait à proximité) était là justement, parmi d'autres.

«Quel est le sens de ce poème?

— Tu sais bien ce que c'est... ce qürärto, c'est à propos de mes ennemis.

- Qui sont tes ennemis ? ». Sans répondre, il détourna les yeux. Me rappelant quelques histoires entendues chez mes hôtes, je tentai le nom d'un de ses voisins. En l'entendant, il se fendit d'un large sourire : «Oui, tu as compris. Tu as bien compris le poème. »

Ce que j'avais compris était que ce chant tenait son origine d'une situation de communication : une insulte adressée à un homme absent de la scène mais peut-être à portée de voix ${ }^{21}$. Il faut dire que Gätənnät était alors en conflit ouvert avec ce voisin arrivé depuis peu au village, suite à un mariage, et qui cherchait, apparemment avec une certaine agressivité, à augmenter ou du moins consolider ses terres. Le poème menaçant de Gätənnät, qui survenait à la suite d'une série d'incidents de gravité croissante (problèmes de bordure, bétail envoyé saccager les récoltes, pressions en faveur d'un divorce, bagarres et coups de fusil tirés) prend

20. Le « wax and gold (səməna wärq), rendu célèbre par Donald Levine (1965) s'appuie sur les similitudes de sonorités pour construire des doubles sens.

21. Ce procédé est apparemment très commun, car il m'a été rapporté à plusieurs reprises. 
alors tout son sens. Il dit quelque chose de leur rivalité et de la tension qu'elle génère, en contraste frappant avec la solidarité qui règne entre la poignée de parents et d'amis fidèles venus ce jour-là aider aux travaux des champs.

J'ai souligné plus haut que la poésie chantée est présente (ou susceptible de l'être) dans toutes les circonstances de la vie sociale, des rituels aux réunions intimes. Elle l'est d'autant plus, comme le montre l'étude détaillée de ces performances (Morand, 2010, 2011), lorsque ces circonstances exigent que l'on démontre soutien et réciprocité ; ce qui ne va jamais de soi dans un système de parenté sans lignage ni groupe fixe, sujet à de constants changements d'alliances et où de sévères conflits peuvent éclater même (et surtout !) entre parents très proches $^{22}$. La poésie chantée, à laquelle chacun est libre de participer ou non, représente alors un degré supplémentaire d'engagement. Les performances y sont à la fois une image des relations personnelles et un outil pour les conforter. Comme le souligna l'un de mes interlocuteurs, « aux funérailles, par exemple, c'est chacun son tour. Si j'ai chanté de l'angurguro aux funérailles d'une personne, ses parents viendront le faire et tireront au fusil lors des funérailles de mon propre parent $\gg^{23}$.

Dans ce contexte, les poèmes, pour être de quelque intérêt, doivent dire « juste » (lakk) et «vrai » (awnät), selon les deux termes les plus fréquemment utilisés pour les qualifier. Autrement dit, ils doivent prendre la réalité - la situation, les faits - pour sujet, et en fournir une perspective jugée adéquate par l'audience. Ces perspectives sont bien sûr des interprétations personnelles, des opinions qui apportent un éclairage sur la situation et les problèmes du moment, sur les conflits et les ennemis, mais aussi sur les tensions sous-jacentes, et parfois depuis longtemps enfouies, qui traversent l'assemblée. Malgré leur style elliptique, ces prises de position se laissent aisément déchiffrer par quiconque en connaît le contexte, et s'accompagnent de réactions très vives de la part des auditeurs : approbations, interjections, coups de fusil et autres manifestations émotionnelles qui, en cas de désaccord, peuvent dégénérer en assauts et en bagarres. Il s'agit là d'un mode de communication qui diffère du langage ordinaire non par ce qu'il s'y dit - mots de soutien et insultes peuvent très bien se passer du chant - mais par sa singulière efficacité rhétorique. Cette dernière réside en partie dans les stratégies verbales et en partie dans les propriétés contrastées des trois genres

22. Une des caractéristiques du système de parenté bilatérale des Amhara est qu'il crée des rivalités directes entre parents proches pour l'accès à la terre, qu'il s'agisse des systèmes agraires d'ancien régime - avant la révolution de 1974 - ou de ceux mis en œuvre plus récemment (НOBEN, 1973 ; MorAND, 2011).

23. Təgab, mars 2007. 
chantés : le zäfän joyeux et aux rythmes enlevés; le qärärto, souvent à la limite de la colère, avec ses grandes envolées enflammées ; et l'angurguro, triste et angoissé, dont la mélodie imite les sanglots (Morand, 2010). Certains chants peuvent avoir des conséquences considérables, comme dans le cas, présenté dans un numéro précédent de cette revue, de l'enterrement d'un jeune homme tué par son propre frère cadet (Morand, 2011).

Le poème chanté par Gätənnät à son enclos aux vaches est donc la trace d'un tel moment de communication, face à une audience pour laquelle il s'efforçait de dire avec « justesse » sa colère. Il ne prend tout son sens que dans cette situation passée; pourquoi alors le répète-t-il en privé des années plus tard ? Parce que le poème peut parfaitement reprendre sens dans le présent. L'inimitié entre Gätənnät et son voisin, après avoir connu plusieurs mois d'accalmie, venait de connaître une nouvelle flambée et la colère - portée par la mélodie du qärärto - semblait s'alimenter dans sa solitude, comme s'il ressassait les récents événements à la lumière du passé. Le chant « privé » existe en tant qu'écho d'une communication qui a déjà eu lieu : une communication différée, un peu à l'instar des longs poèmes jamooje na'i déclamés par les bergers peuls du Mâssina lors de leurs transhumances solitaires et dont Christiane Seydou (1991) nous explique qu'ils sont une projection vers des performances futures. Pour les Peuls, l'interaction est à venir ; pour les Amhara du Goğğam, elle appartient au passé. Dans les deux cas, la performance s'appuie sur l'imagination pour pourvoir à ce qui dans la solitude fait défaut : des destinataires.

À qui s’adresse donc Gätənnät ? Plusieurs scénarios sont envisageables. On pourrait spéculer qu'il :

- reproduit en imagination la situation de communication passée et adresse, pour une seconde fois, son chant à ces auditeurs d'alors, auditeurs dont il aura certainement besoin dans les nouvelles difficultés qui s'annoncent (en particulier son jeune frère, couché de l'autre côté de l'enclos);

- engage son ennemi, comme si ce dernier pouvait entendre le sérieux de sa nouvelle menace ;

- s'adresse à lui-même au présent en prenant son soi passé à témoin ou vice versa.

Probablement s'agit-il d'un peu tout à la fois : un discours aux interlocuteurs imaginés qui mêle au présent un passé revu et réinterprété. Ce type de performance ressemble d'ailleurs beaucoup à ce qu'on appelle le « dialogue intérieur ${ }^{24}$, ce discours pour soi-même qui peut être aussi bien silencieux que vocalisé. Celui-ci

24. En anglais « inner speech » ou « self talk », les théoriciens ne s'accordant pas tous sur leurs définitions. 
fut longtemps considéré comme pathologique, mais depuis la redécouverte des travaux de Lev Semenovich Vygotsky (1962), qui le considérait comme une internalisation des interactions sociales, de plus en plus d'auteurs mettent en avant l'aspect régulateur de ce phénomène (Emerson, 1983). Pour le psychologue Alain Morin, ce «self talk » comprend aussi bien le dialogue avec des personnes fictives que le dialogue avec soi-même : permettant d'expérimenter les perspectives des autres, il joue un rôle dans les émotions et les réactions, et favorise la réflexivité et la construction du soi (Morin, 1993). Gätənnät, ravivant son accès de colère, et Haymanot, pleurant sur ses soucis en revoyant l'enterrement de son père assassiné, sont certes seuls, mais pas entièrement à eux-mêmes. Parents, voisins, alliés et ennemis les y suivent en un dialogue sans cesse réimaginé.

\section{Conclusion}

C'est donc une certaine densité de la solitude que nous montre ce parcours dans les musiques privées des paysans Goğğamés. La solitude, nous l'avons vu, n’est pas entièrement coupée du monde social ; et, bien qu'elle soit le plus souvent définie par ce qu'elle n'est pas - c'est-à-dire la sociabilité - elle n'en est certainement pas une échappatoire. Elle est en premier lieu culturellement définie : ce que sont ces lieux et temps, et ce qu'être seul (ou, dans notre cas, seul en compagnie) veut dire est déterminé par les institutions sociales que sont le calendrier religieux ou agricole, la parenté et la structure des échanges en général. Ces représentations et ces expériences devraient à leur tour permettre d'éclairer, malgré les difficultés de l'ethnographie, l'intersection entre individu et société dans une culture donnée.

Chants et paroles solitaires en sont peut-être l'une des meilleures fenêtres. Leur fonction n'est pas entièrement claire : pour C. Seydou (1991), elle serait d'éviter le silence et de rester dans le social, c'est-à-dire de préserver sa propre humanité. Ce qui est certain, c'est que pour les Amhara du Goğğam, ces chants naviguent entre le réconfort et l'intrusion. Ces dialogues réimaginés et réinterprétés ressemblent à une version plus élaborée, et surtout plus puissante émotionnellement, des bribes de conversations imaginaires qui au quotidien se bousculent à l'esprit : ils convoquent passé, incertitudes présentes et peut-être décisions à prendre. Au bout de quelques mois, on commençait à me poser des questions. On cherchait à savoir ce qu'un parent ou un voisin avait chanté la veille dans la solitude : de quels genres, quels poèmes, et quelles manifestations émotionnelles j'avais été témoin. Le privé semblait, après tout, un peu l'affaire de tous. 


\section{Bibliographie}

Selon l'usage, les auteurs éthiopiens sont indexés par leur prénom, suivi du prénom de leur père.

Ashenafi Kebede, 1971, The Music of Ethiopia: its Development and Cultural Setting, thèse de Doctorat, Wesleyan University.

BAETEMAN Joseph, 1929, Dictionnaire amarigna-français suivi d'un vocabulaire français-amarigna, Dire Dawa : Imprimerie Saint-Lazare.

Casajus Dominique, 2000, Gens de parole : langage, poésie et politique en pays touareg, Paris : la Découverte.

Cohen Marcel, 1924, Couplets amhariques du Choa, Paris : Imprimerie nationale.

Clastres Pierre, 1974, la Société contre l'État, Paris : Éditions de Minuit.

Delombera Negga, 2004, « Tezeta, le Souvenir et la Nostalgie », in Michel Demeuldre (dir.), Sentiments doux-amers dans les musiques du monde, Paris : l'Harmattan, p. 33-41.

Emerson Caryl, 1983, “The Outer Word and Inner Speech: Bakhtin, Vygotsky, and the Internalization of Language”, Critical Inquiry, vol. 10, n 2, p. 245-264.

FAlCEto Francis, 2004, « Tezeta dans la musique populaire éthiopienne », in Michel Demeuldre (dir.), Sentiments doux-amers dans les musiques du monde, Paris : l'Harmattan, p. 43-44.

Finnegan Ruth, 2011, Why Do We Quote? The Culture and History of Quotation, Cambridge: Open Book Publishers.

Getie Gelaye, 2000, Peasants and the Ethiopian State: Agricultural Producers' Cooperatives and their Reflections in Amharic Oral Poetry, Hamburg: LIT.

Hoben Allan, 1973, Land Tenure among the Ambara of Ethiopia: The Dynamics of Cognatic Descent, Chicago: the University of Chicago Press. 
Kane Thomas, 1990, Ambaric-English Dictionary, 2 vol., Wiesbaden: Harrassowitz.

Levine Donald, 1965, Wax and Gold, Tradition and Innovation in Ethiopian Culture, Chicago: the University of Chicago Press.

Lortat-JaCoв Bernard, 1998, Chants depassion, Paris : Éditions du Cerf.

Lortat-Jacoв Bernard, 2002, « les Faits musicaux ne sont pas des choses », in Jean-Marc Chouvel et Fabien LÉvy (dir.), Peut-on parler d'Art avec les outils de la Science?, Paris : l'Harmattan, « Cahiers de l'Ircam », p. 451-480.

Menghistu Lemma, 1963, « Yäamarəña gəțəm aynätu səratu sərəatu », Journal of ethiopian studies, vol. 1, n² 2, p. 133-151.

Mondon-Vidailhet Casimir, 2003 [1922], « la Musique éthiopienne », Annales d'Éthiopie, no 19 , p. 149-187.

Morand Katell, 2010, «Vaine Existence : entre deux genres, ou les effets d'un poème chanté », in C. Calame, F. Dupont, B. Lortat-Jacob, M. Manca (dir.), La voix actée, Pour une nouvelle ethnopoétique, Paris ; Éditions Kimè, p. 23-41.

Morand Katell, 2011, « Mélodies pleurées, paroles à attraper : les chants d'angurguro dans des funérailles éthiopiennes », Cabiers de Littérature Orale, $n^{\circ} 69$, p. 57-78.

Morand Katell, 2013, «En forêt, la musique : entre inquiétude et sentiment d'intimité (Goğğam, Ethiopie) », Etnográfica, vol. 17, n 3, p. 561-579.

Morin Alain, 1993, "Self-talk and Self Awareness: On the Nature of the Relation”, The Journal of Mind and Behavior, vol. 14, $\mathrm{n}^{\circ}$ 3, p. 223-234.

Rouget Gilbert, 1980, la Musique et la Transe, Paris : Gallimard.

RousseAu Jean-Jacques, 1983 [1778], les Rêveries du promeneur solitaire, Paris : le Livre de Poche. 
Seydou Christiane, 1991, Bergers des mots : poésie peule du Mâssina, Issy-lesMoulineaux : les Belles Lettres, « Classiques africains ».

Taylor Anne Christine, Chau Ernesto, 1983, “Jivaroan Magical Songs, Achuar Anent of Connubial Love”, Amerindia, n ${ }^{\circ}$, p. 87-127.

Vygotsky Lev Semenovich, 1962 [1934], Thought and Language, Cambridge: The M.I.T. Press.

Weisser Stéphanie, 2008, « le Bagana, instrument de musique et de prière amhara », Annales d'Éthiopie, no 23, p. 61-76.

Weisser Stéphanie, Falceto Francis, 2013, "Investigating Qəñət in Amhara secular music: an acoustic and historical study", Annales d'Ethiopie, $\mathrm{n}^{\circ} 28$, p. 299-322.

Résumé : Cet article porte sur la pratique dite privée du chant chez les paysans Amhara du Goğğam, en Éthiopie. Il se penche sur ce qu'il se passe dans les interstices de la vie sociale, lorsque fêtes et commémorations sont passées et que les individus se retrouvent seuls avec eux-mêmes : que chantent-ils, pourquoi, et quel est le statut énonciatif de ces performances sans public ? L'analyse des discours et des poèmes chantés est combinée à une ethnographie de la sphère privée pour saisir la place de ces chants dans l'univers musical des Amhara. Dialogues imaginés et sans cesse réinterprétés, ils n'existent, on le comprend, que par référence à un monde social dont la solitude ne peut être l'échappatoire.

Mots clés : anthropologie, musique, poésie, Éthiopie, Amhara, solitude

Abstract: This article discusses the practice of singing in private among the Amhara peasants of Goğğam, in Ethiopia. It investigates what happens in the interstices of social life, when parties and commemorations are over and individuals find themselves alone: what do they sing, why and what is the enunciative status of these performances without audience? The analysis of discourses and sung poems is combined with an ethnography of privacy to uncover what position these songs occupy in the musical world of the Ambara. As imagined as constantly reinterpreted dialogues, they only exist with reference to a social world solitude cannot help escape.

Keywords: Anthropology, Music, Poetry, Ethiopia, Ambara, Solitude 